\title{
Erratum
}

\section{Usefulness of the Electrocardiogram in Diagnosing Mechanisms of Tachycardia}

\section{M.A. Tipple}

British Columbia Children's Hospital, Vancouver, British Columbia, Canada

\section{Re: Pediatr Cardiol 21:516-521, 2000}

In the printed version of this article, unfortunately, the final figures and captions were not presented correctly. The correct version of Figures 7-10 are presented below.

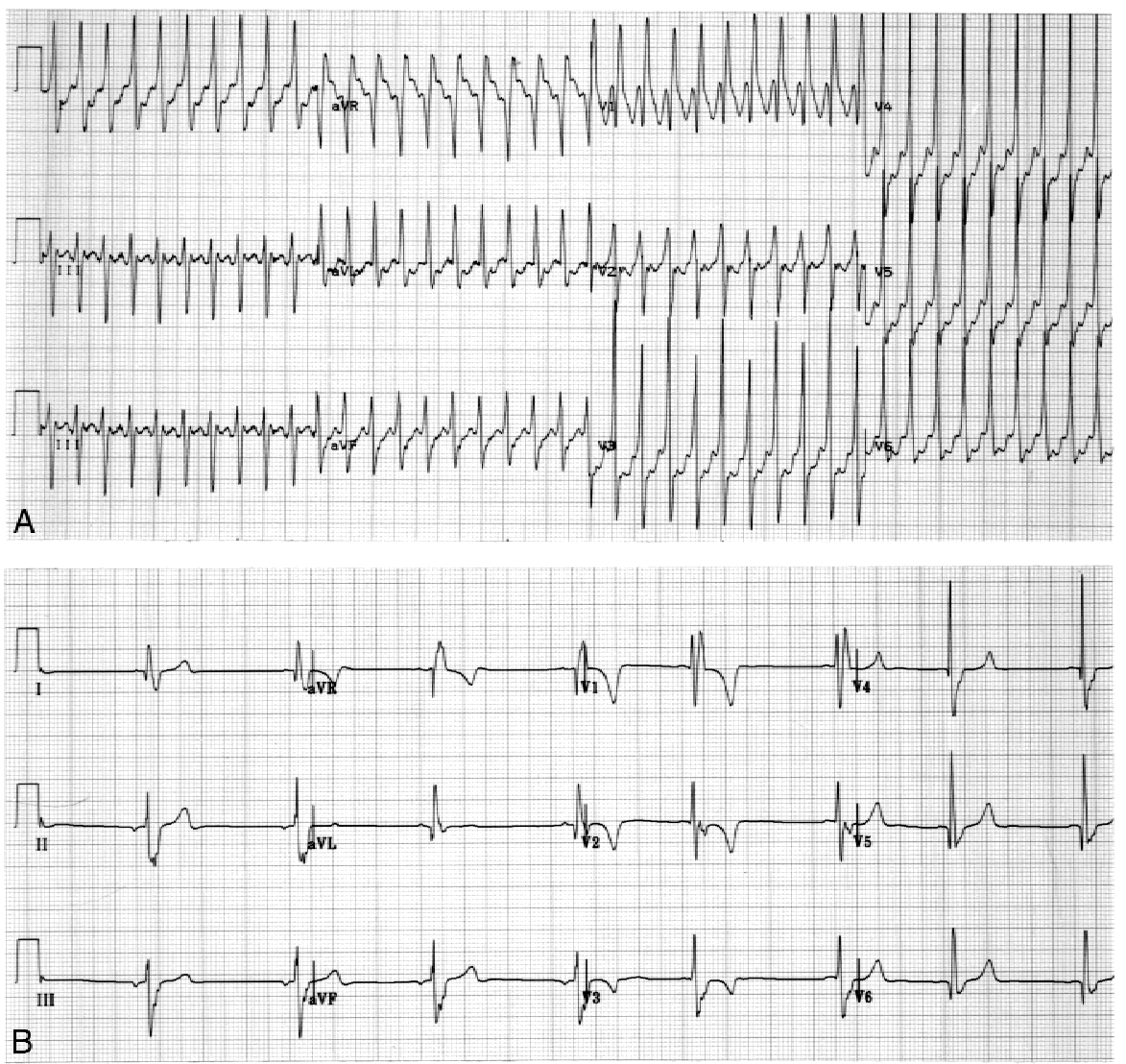

Fig. 7. (A) Wide complex QRS tachycardia 244 beats per minute. RS pattern $\mathrm{V}_{2-6}$. RBBB morphology with 1:1 VA conduction (retrograde $P$ waves visible in ST segment following each QRS). (B) Same patient in sinus rhythm showing RBBB morphology identical to that of the tachycardia. Ten-year-old child postventricular septal defect repair. 

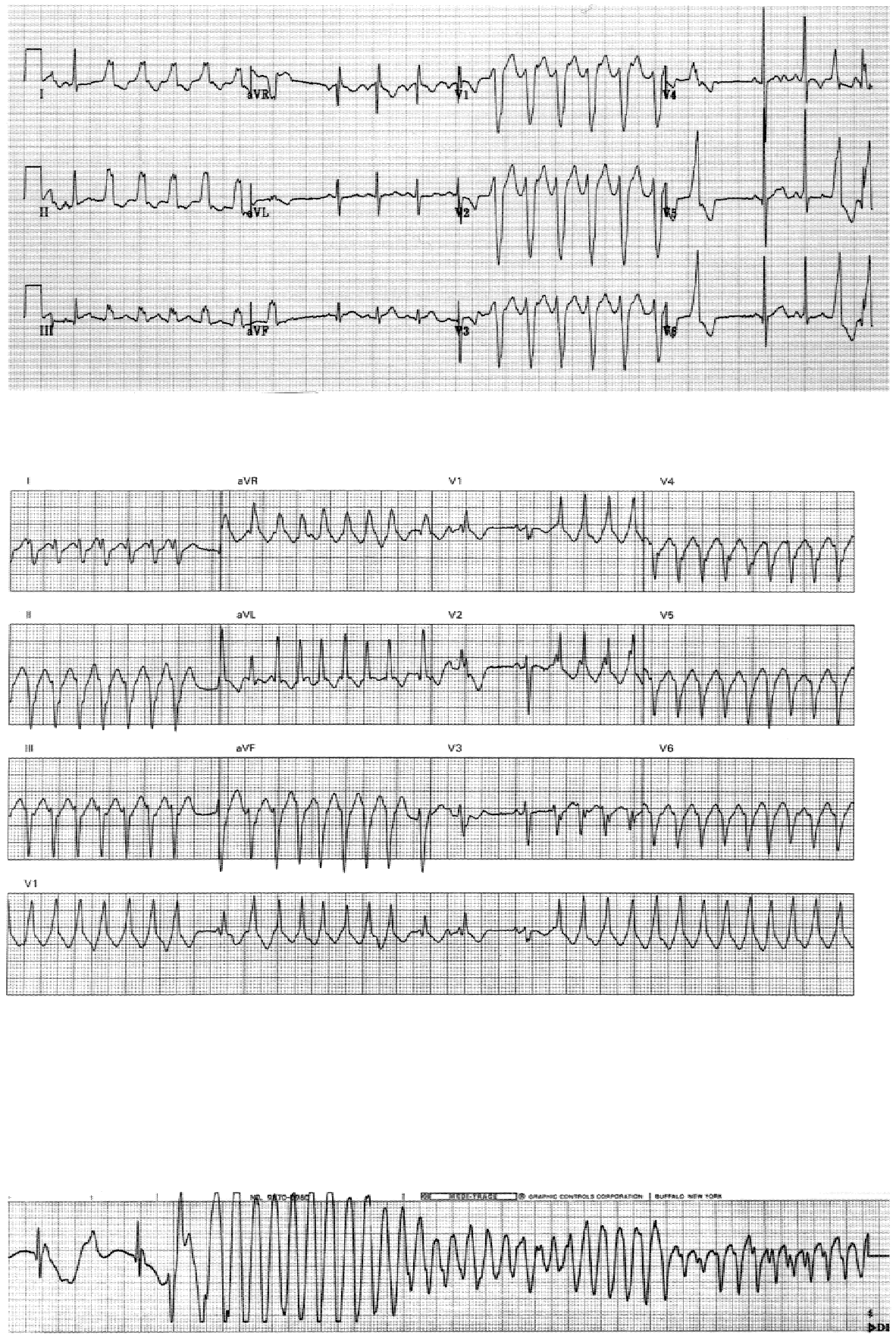

Fig. 8. A 1-year-old child with intermittent, accelerated ventricular rhythm. Rate is only slightly above sinus, RS pattern is present but RS interval is $>100 \mathrm{msec}$, and VA dissociation is present.

Fig. 9. Ventricular tachycardia in a 13 -year-old with a history of exercise-induced syncope. RS pattern is absent, and VA dissociation and fusion beats are present. The tachycardia was ablated in the mid-lower septal region of the left ventricle.

Fig. 10. A 17-year-old with dilated cardiomyopathy and complete heart block undergoing AICD implantation. Rhythm strip showing first junctional beat with prolonged, morphologically abnormal ST-T wave. An afterdepolarization occurs following the second beat, triggering polymorphic ventricular tachycardia. 